\title{
A Study on the Construction and Utilization of Korean Prehistoric Remains Database
}

\author{
Heesoo Choi, ${ }^{1}$ Jong Youl Hong, ${ }^{2}$ Sangheon Kim, ${ }^{1}$ and Kwangcheol Rim ${ }^{3}{ }^{3}$ \\ ${ }^{1}$ Department of Historical Contents, SangMyung University, 20 Hongjimun-2gil, Jongrogu, 03016, Seoul, Republic of Korea \\ ${ }^{2}$ Department of Culture Contents, Korea University, 2511 Sejong-ro, Sejong 30019, Republic of Korea \\ ${ }^{3}$ The Basic Science Institute, Chosun University, 309 Pilmundae-ro, Dong-gu, Gwangju 61452, Republic of Korea
}

Correspondence should be addressed to Kwangcheol Rim; rim1201@hanmail.net

Received 13 October 2021; Revised 17 November 2021; Accepted 10 December 2021; Published 27 January 2022

Academic Editor: Ahmed Farouk

Copyright $\odot 2022$ Heesoo Choi et al. This is an open access article distributed under the Creative Commons Attribution License, which permits unrestricted use, distribution, and reproduction in any medium, provided the original work is properly cited.

\begin{abstract}
The excavation of the prehistoric sites in Korea has been on since the Japanese colonial period. However, it was only after the 1970s that it was undertaken in earnest. Many excavation research institutes, including state agencies, are still conducting excavation research. However, the excavation report, which summarizes the findings, is not serviced on an integrated platform. As a result, acquisition of integrated knowledge and research on Korea's prehistoric remains are not properly facilitated. Therefore, it is urgent to establish a database of prehistoric remains. This requires considering the characteristics of archeological excavation work and the specificity of publishing excavation reports. It is desirable to design and build database (DB) tables for excavation reports of relics and ruins, multimedia, and excavation investigations, by focusing on the DB table for prehistoric remains. Once the database is established, it will help expand cultural heritage information services through tools, such as electronic maps or the Internet of Things.
\end{abstract}

\section{Introduction}

1.1. The Necessity of Building a Database of Information on Prehistoric Remains in Korea. In historical research, the oldest historical sites are fascinating to explore. This is because they represent history of the present and help explore the present time's origins. In particular, the exploration of the period before the written script is entirely dependent on the achievements of archeological excavations [1]. Therefore, the systematic service of excavation reports, the result of excavations of prehistoric sites, can be of great help for historical research.

The excavation of remains of the Korean prehistoric period began in the era of Japanese occupation. The investigation by Japanese scholars strongly distorted results and denied the long history of the Korean Peninsula. After independence from the Japanese, the excavations carried out by Koreans were aimed at correcting history that was ignored or intentionally distorted by Japanese scholars. As a result, many remains of the prehistoric times, dating back to the period spanning the Paleolithic Age to the Bronze Age, have been found on the Korean Peninsula, as in other regions.

Initially, the main subjects in charge of the excavation were the national research institutes. However, as the discovery of the remains progressed, university research institutes and private research institutes in the region gradually became involved. These phenomena, and further academic development in the field of archeology and active research activities on historical sites, were perceived as a positive development. However, it is difficult to obtain comprehensive information on prehistoric remains. For instance, the excavation reports prepared by various research institutions are neither systematically organized nor accessible. Instead, they are separately managed and provided by each institution, or through commercial services offered by private companies.

Among the information on prehistoric remains, crucial information pertains to the period to which the prehistoric remains belong, the characteristics of the space it is located 
in, and the other relics found in these ruins. Furthermore, it is important to study the history of the relevant era, such as that of how the ruins are distributed across the Korean peninsula, by synthesizing such crucial pieces of information. Therefore, a systematic information service for prehistoric remains needs to be urgently established.

However, this study had some limitations. First, the information related to prehistoric remains is not integrated online or offline but is scattered. Therefore, it is necessary to provide information, on a unified platform. Second, the various materials related to prehistoric sites, such as booklets, digitized files, photographs, drawings, and images, are not systematically managed. Most of them are found in excavation reports of excavating organizations, but digital files such as images and 3D objects cannot be viewed in the report. In other words, it is necessary to manage digital information regarding excavations systematically. Third, various information related to prehistoric remains are not provided in many formats.

Therefore, it is urgent to establish a database of prehistoric remains. In this paper, we review the requirements for building a database of prehistoric remains and attempt to construct a DB table for building a database that is based on the results of the review. Furthermore, we would like to explore ways to utilize the database of prehistoric remains as a "knowledge platform" [2].

\section{Materials and Methods}

2.1. Current Status of Korean Prehistoric Remains Excavation Report Service. Among the information on prehistoric remains in Korea, the most significant is the excavation report. The excavation report has a rich stock of information on aspects such as the various appearances of the remains in the excavated sites, the existence of the relics excavated from the ruins, and the historical significance of the remains. Therefore, the excavation report can be said to be the most basic set of data on prehistoric remains in Korea. What are the ways to obtain excavation reports? The excavation report service of the remains investigated in Korea has recently been provided through the Internet as administrative information. The representative example is the excavation investigation report service provided by the Cultural Heritage Administration [3]. (Figure 1).

This service provides a PDF file of excavation reports submitted in Korea from July 8, 2005, to December 31, 2020. A total of 4,724 excavation reports have been uploaded, and the desired information can be obtained by selecting the name of the site, publishing agency, the number of investigation attempts, and year of submission. By default, it provides reports from the latest submission date. To find the information, it is desirable to use the search results after entering the name of the site or the investigation area.

As previously mentioned, the core elements of information related to the remains are era, space, and relics. However, the previously mentioned service only provides information regarding space, that is, location-related information. For example, the methods used to check timerelated information, such as the Paleolithic Era and
Neolithic Era, can only be understood by reading the excavation report.

The National Research Institute of Cultural Heritage also provides excavation reports through the original text information service of the research knowledge portal on cultural heritage [4]. When searching for a given excavation report, only 87 cases appeared. If the excavation survey was, on the other hand, selected by the chronology of the excavations, a total of 15,378 excavations were found. Thus, the deviation seems to be very severe. However, it ought to be considered that excavation-related documents such as academic symposium data books and other original texts also appear in the search results. Thus, the actual number of excavation reports is much smaller.

Similar to the case of the Cultural Heritage Administration, the main information provided by the National Research Institute of Cultural Heritage is very poor. Fortunately, its service is easier to use than the Cultural Heritage Administration's Service because it provides an abstract service that helps find key information. However, it is also necessary to read the original text, in PDF, to properly comprehend information by region (space), age (time), or relic-related parameters (Figure 2).

Museums, one of the major organizations overseeing excavations, also provide information-related services. A representative example is the excavation report service of the National Museum of Korea [5]. This museum provides its own reports on excavations and investigations (Figure 3 ).

This online service does not conveniently provide appropriate information about the remains. The title and author of reports are the main service standards, while information on the period, region, and major relics can be viewed by downloading the report's PDF file. Only a detailed table of the contents of the report is organized and provided. Moreover, some reports are for sale in cultural goods stores. Thus, not all reports are available online.

As previously described, this study briefly reviews the major excavation report services in Korea. As a result, it was found that excavation report services have several problems. First, it is difficult to find the desired excavation report easily because there are various types of organizations and services that provide such reports. Although the services of major national institutions were examined, there are many other services given by private institutions where each of them provides their own published reports in various ways, thus, making it difficult for researchers to find the information they want.

Second, the contents of the service are concentrated only in the book called "Excavation Report." In the case of the National Museum of Korea, bibliographic information is faithfully provided, but the contents of an important excavation report cannot be known unless the original text has been read. The same applies to the rest of the services. The location-related information of the ruins can be found because it appears as a place name in the title of the report, but other information is hidden. In particular, information on the relevant period and major relics of the remains is not revealed.

Owing to these issues, it is urgent to establish a plan to integrate the excavation reports into a single service. 


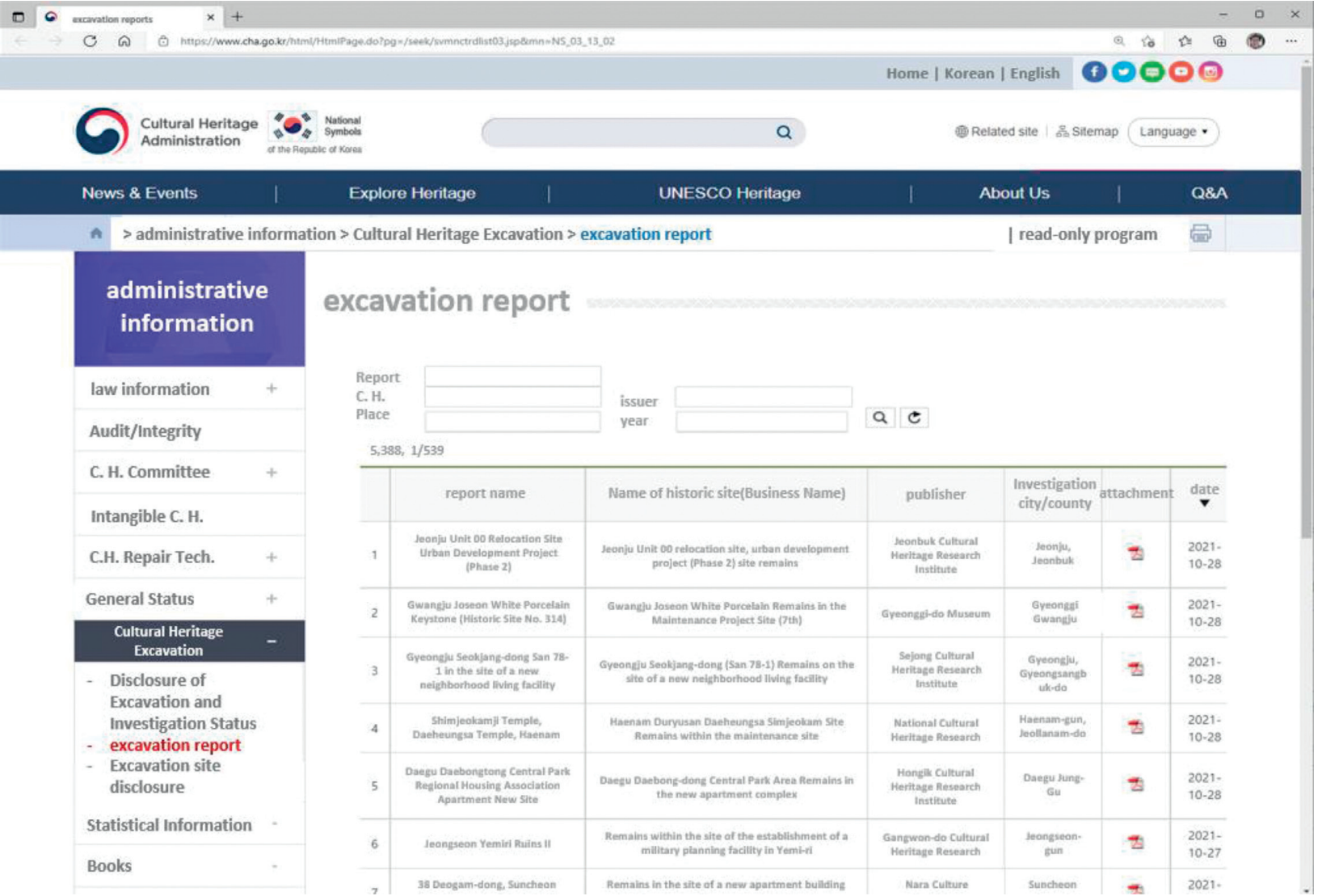

Figure 1: A screenshot of Cultural Heritage Administration's Excavation Investigation Report Service.

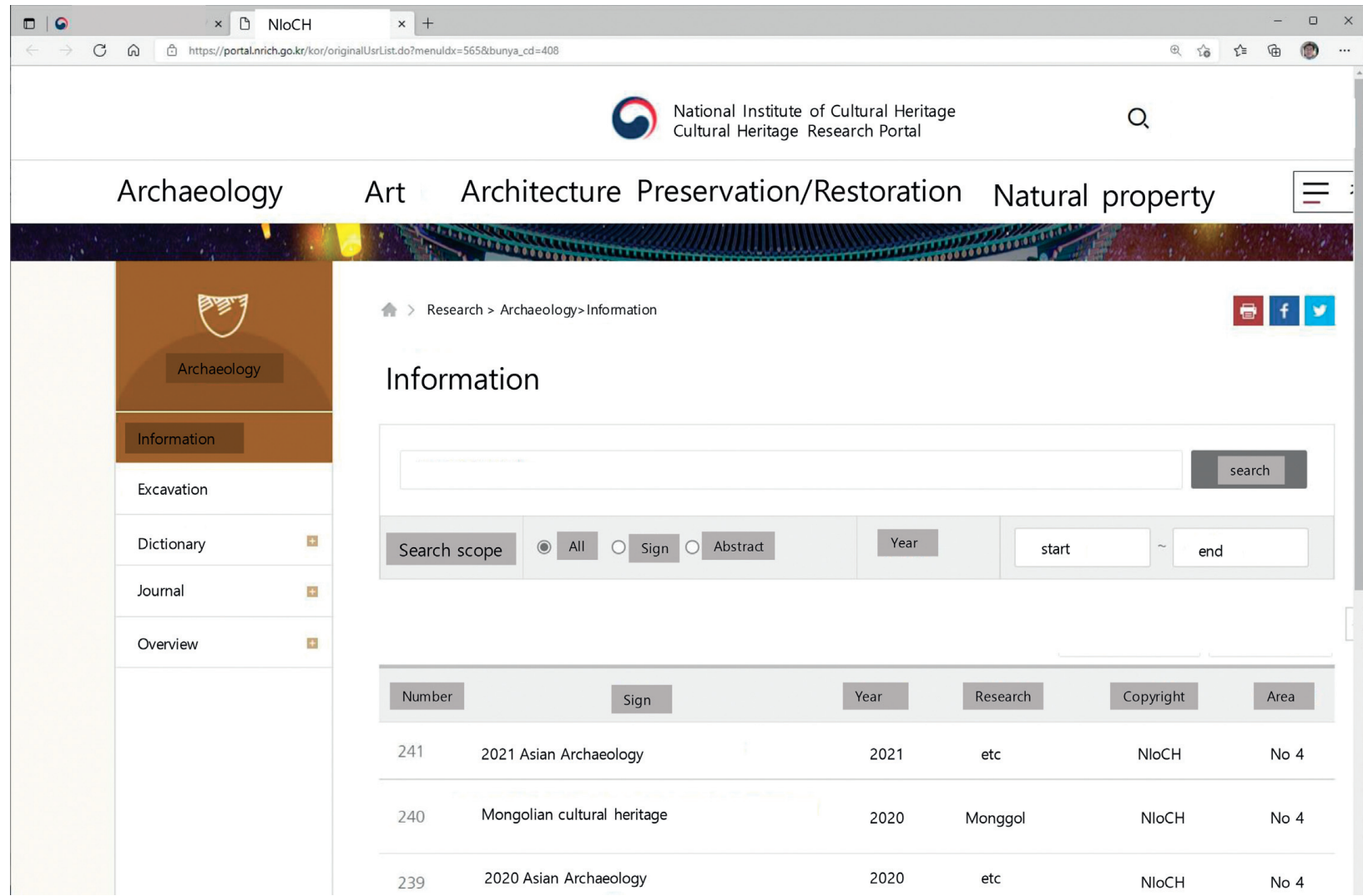

Figure 2: A screenshot of the original text information service of the National Research Institute of Cultural Heritage. 


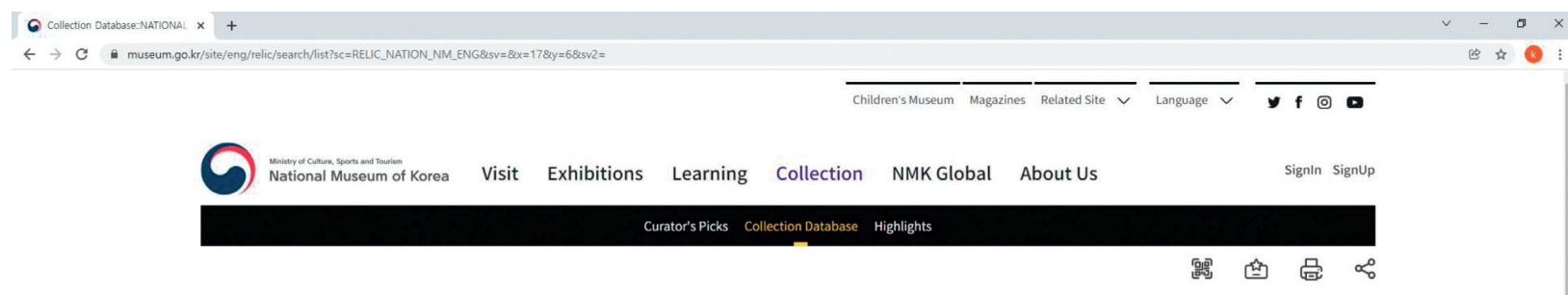

๑ > Collection > Collection Database

\section{Collection Database}

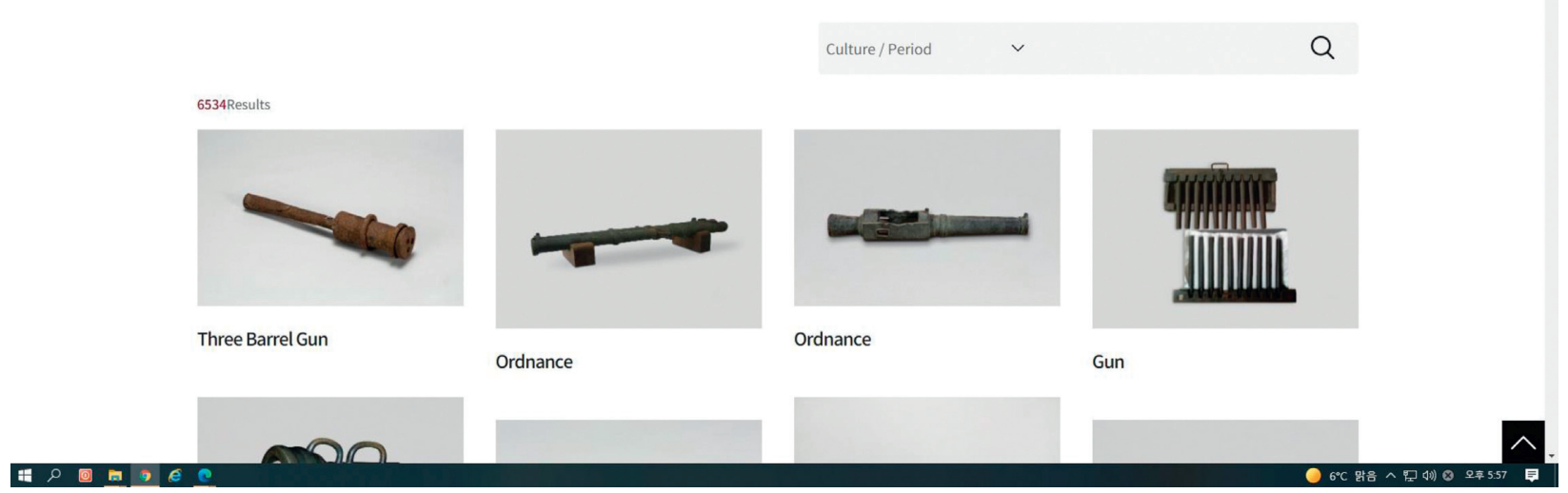

Figure 3: A screenshot of the National Museum of Korea's Excavation Report Service.

Excavation reports are composed by a wide variety of investigative agencies with a variety of contents and methods. Therefore, it is not easy to build standardized databases, such as those of academic papers. Since the specificity of the excavation report must be considered, it is necessary to review the issues of publication of excavation reports.

\subsection{Characteristics of the Excavation of Korean Prehistoric} Remains and the Publication of Excavation Reports. The excavation report is an academic version of the achievement of archeological research. Archeology is a study that contributes to the exploration and restoration of the history of a given time through the remains and relics left by people of that time, in addition to the history of these people, recorded as textual data. There are various objects that need to be researched in archeology, but most of them are buried. The buried objects cannot be identified before excavation; therefore, excavations are considered very important in archeology. Consequently, excavation reports containing archeological findings are very important for restoring the history and culture of a given time. To build a database of excavation reports, it is necessary to consider the characteristics, contents, and methods of archeological research [6].

Several conditions exist as prerequisites for discovering unknown archeological data. First, it should be possible to estimate that the target area will have archeological data. It is not possible to randomly excavate anywhere, so evidence is needed to gauge that there will be archeological data in the given area, through an examination of characteristics such as historical and cultural traces around it, or historical records. Second, even if there is evidence of archeological data in an area, several processes must be undertaken to determine the importance of this data. Since it is difficult to dig up an entire area, it is necessary to find data on a trial basis or investigate it through several stages [7].

Archeological excavation research involves several stages. First, excavation is carried out after a surface survey was done to collect data. A surface survey is conducted to find traces of relics or remains exposed to the surface before conducting a full-scale survey of buried materials. If an indicator survey determines that an excavation survey is necessary, an area believed to be an intensive distribution area for relics will be excavated. Excavations will also be conducted first to check the status of the buried materials before starting a full-scale excavation investigation.

Archeological excavations are conducted considering several purposes. First, there is an ordinary excavation conducted by archeologists. Then, there is research excavation that involves conducting research to verify hypotheses established for certain kinds of remains. Third, rescue excavation accounts for the largest portion of the excavation surveys. Rescue excavations should be conducted in areas at risk of destruction due to natural collapse of the ruins or manmade civil engineering structures. If a full-fledged excavation investigation is required after ordinary excavations or rescue excavations, research excavation can be conducted for decades. In other words, excavations at a site can be carried out several times.

As previously shown, the excavation process progresses, while the excavation research is summarized. Excavation is the process of collecting and recording archeological data. Through this process, the data collected are analyzed and interpreted. The data collected during the excavation survey are classified by type, their age measured, raw materials, and 
functions identified, and, subsequently, they are compared with other sites. This is an analysis of archeological data. This process also involves a connection between data and cultural interpretation.

When the analysis is conducted, the intellectual process of extracting meaningful content from archeological data is carried out, which is an interpretation. There are two main interpretations of this study. One is the cultural-historical interpretation. This is used to discover the temporal, spatial, and functional elements of the site. The other is the interpretation of cultural processes. This involves the task of tracking changes in culture. The work that archeologists conduct through excavation research is to identify the nature of the remains and relics [8].

Various contents are recorded during excavation surveys. Above all, it is important to find an oil field that has traces of past structures, through the excavation of historical sites and recover and record the ruins. To begin excavating, the target area is selected and divided into several excavation sections, and excavation is carried out sequentially for each section. The data obtained during this process is recorded and collected by archeologists. The materials found are stored after systematically recording the relics in the same sequence as they were excavated, thus giving them their original location and serial number.

This is a brief overview of the characteristics and contents of archeological excavations. It is an excavation report that contains the core contents of the excavation investigation [9]. Therefore, some points from an excavation report can be summarized to build a database of excavation reports. First, the excavation report can be classified in various ways depending on the purpose and content of the excavation. Excavations are divided into categories such as ordinary excavations, research excavations, and rescue excavations. It is also confirmed that all excavations can be classified into surface surveys and excavation surveys and that excavation surveys are conducted several times depending on the characteristics of the site. Therefore, there may be several excavation reports at one site, and each excavation report may be divided according to its purpose and content.

Second, the excavation report contains various contents related to the excavation survey. Once the investigation is decided upon, an investigation team will be formed. This team comprises institutions selected as excavation agencies, and the head of the investigation team and investigators. Excavations by the investigation team usually take place over a fixed period of time. In general, one excavation report is based on a single purpose of investigation. This is closely related to the purpose of the excavation. In addition, information related to the publication of the excavation report is included after the excavation survey.

Third, the excavation report contains details of the excavation survey. In fact, these form the most important part of an excavation report. The analysis and interpretation of the archeological data previously discussed are determined using these details that largely pertain to geographical information of the site, remains excavated from the site, and relics excavated from the site.

Information on the remaining site is mainly geographical, including information such as location and geographical environment relating to the natural environment. Additionally, it also included information such as history, culture, and cultural heritage of the surrounding area related to the manmade environment. It can be accompanied by various drawings, such as geological maps, topographical maps, location maps, and distribution charts.

The remains refer to traces of past structures examined at the site. These include building sites such as houses and defensive facilities such as fortresses, traces of walls, and tombs. The shape, size, and structure of the ruins are very important because they can help identify the era to which the remains belong and various cultural phenomena. The characteristics of the site can also be defined by ruins. It can be classified as a residential site such as a house, or a village site. It can also be classified as a defensive site through identification of traces of defense facilities, or a religious site such as a Buddhist temple site, or a burial site such as a tomb, or a comprehensive site with various traces found (Figure 4).

Relics are the most carefully approached materials when examining remains. This is because they have been buried for a long time and are most vulnerable to damage when exposed. Relics can be objects people used in their daily lives. This is why all the data believed to be there can be excavated. Relics considered important in cultural history and cultural processes in archeological research so far include earthenware, stone tools, bronze weapons, and bronze ritual tools. Their excavation status is also very important for interpreting the findings of excavations.

To build a database of excavation reports on Korea's prehistoric remains, these points must be reflected upon. However, there is something to be considered here. The title is the most basic information of the excavation report. The title is determined by the purpose and scope of the excavation report. However, only after the excavation survey is completed, the value of the site established, and the site acquired as a cultural heritage site, is the name of the remains determined.

For this reason, the title of the excavation report often does not include the names of the remains known. In other words, the title makes it difficult to find information about historical sites. For example, in the case of the Jeonggwandong remains in Busan, the titles of excavation reports are "Cultural Remains Survey Report-Gijang-gun [10]," "Gadong, Gijang Historic Site in Jeonggwan New Town Development Zone in Busan [11]," and "Cultural Remains Research Institute in Yongsu-ri, Jeonggwan-eup, Gijang-gun [12]." The remains of Jeonggwan-dong are called Yongsu-ri Gadong Historic Site and Gijang Bangok-ri Historic Site. Thus, it is not easy to find information on the Jeonggwandong ruins in these titles.

It is desirable to use titles, of historical sites, that are known to the public, but most of the remains do not have such titles. In particular, in the case of excavation surveys conducted for the purpose of rescue excavation, detailed and effective policies are needed because the local names, addresses, and numbers of the areas are subject to the titles of excavation reports.

We examined the features and contents of the excavation report in Korea and found some problems. For Korea's 


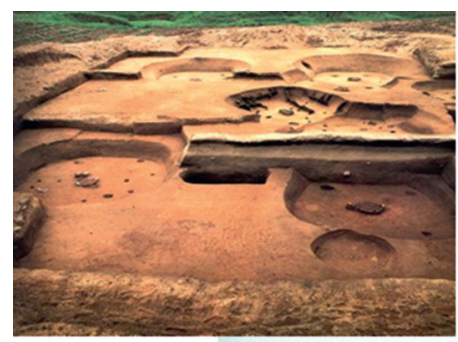

Residential Site in Amsa-dong, Seoul

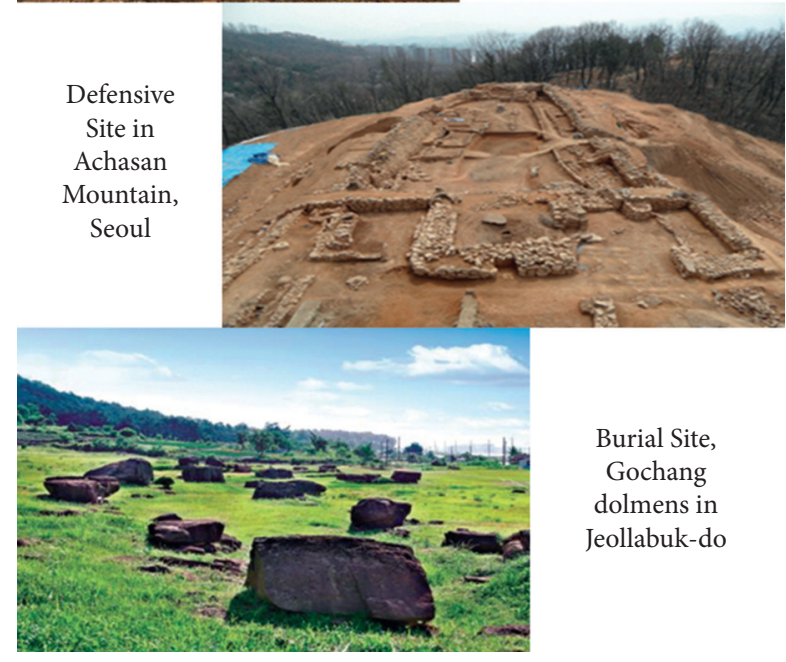

FIgURE 4: Case images of different archeological sites.

prehistoric remains database to be properly built and maintained, it must be first designed. In conjunction with the prehistoric heritage $\mathrm{DB}$, the excavation reports $\mathrm{DB}$, ruins $\mathrm{DB}$, and relics $\mathrm{DB}$ need to be designed separately. In addition, various multimedia data related to excavation research should be connected to the DB. In addition, the establishment of the excavation investigation agency DB will facilitate the establishment of a database that covers various types of information in the archeological field.

\section{Results and Discussion}

3.1. Design of the Prehistoric Remains Database in Korea. As shown in the previous chapter, Korea's prehistoric remains database can be designed with six DB tables. Let us consider what is included in the database field for each table by beginning with the DB table for prehistoric remains.

The table on prehistoric remains contains four main types of information. First, it contains information regarding the physical environment of the remains. These include the site's name, corresponding era, type, and location and information on the excavation report and literature data. In addition, there is information related to excavation surveys, including the investigation agency, composition of the investigation team (team leader, investigator), and the investigation's period, purpose, scope, and method involved.

Information related to the site's investigation is included. It can be largely divided into information, and multimedia, on ruins and relics, including their names and descriptions containing the era, use, structure, and characteristics of the two classes of remains, respectively.
Multimedia information on the remains includes maps such as geological and topographical ones, distribution of historical sites, actual measurements, and photographic, video, and $3 \mathrm{D}$ information (to provide stereoscopic information). Based on this, the prehistoric remains DB table is as follows (Table 1).

Much of what is included in the prehistoric remains information table is extracted from the excavation report. This is why information on excavation reports is important for building the prehistoric remains information DB. As previously mentioned, several excavation reports may exist on one site. Therefore, it is desirable to configure a separate $\mathrm{DB}$ because it is difficult to include all excavation reports in the prehistoric remains information DB.

Since the excavation report is a booklet, it is common to include bibliographical information in it. It also includes the title of the excavation report, the author, the issuing agency, the date of issue, the size (including the number of faces), type of survey, format, language, survey period, table of contents, and overview. The DB table for the excavation report is as follows (Table 2).

Excavations of prehistoric remains should also consist of separate DB tables. In some cases, a single set of ruins is found in prehistoric sites, but most of them are found as a group of several or various types of ruins. The ruins DB table includes the name, era, purpose, structure, characteristics, area, information on excavated relics, excavation reports, and multimedia information. The ruins DB table is organized as follows (Table 3 ).

Similar to the ruins DB table, a relics DB table should be constructed. This is because most artifacts excavated are of many different types. The relics DB table includes the name, era, purpose, structure, characteristics, area, relevant ruins information, excavation report, multimedia information, and so on. The relics DB table is organized as follows (Table 4).

Multimedia information related to prehistoric remains includes all the images contained in the excavation reports of the past. However, owing to recent advances in digital technology, various forms of media, such as maps, drawings, photographs, images, and $3 \mathrm{D}$ objects, are recorded and preserved separately. Therefore, these are very important for prehistoric remains databases. Since a number of different multimedia are produced in relation to one relic or one ruins field, it is necessary to organize a multimedia information DB table separately. The DB table for multimedia information is organized as follows (Table 5).

Finally, DB tables related to the investigation agency must be constructed. In fact, in addition to state institutions, museums at each university and private research institute related to cultural assets are conducting excavations in Korea. Thus, information on these institutes is also needed. The DB table of investigation institutions includes the name, site, investigation's subject, period, and purpose, composition of investigation team, excavation report, and so on. The $\mathrm{DB}$ table of the investigative agency is organized as follows (Table 6).

The six tables previously mentioned are interlinked with the tables that comprise Korea's prehistoric remains 
TABle 1: Prehistoric remains DB table.

\begin{tabular}{|c|c|c|c|}
\hline Elements & References & Elements & References \\
\hline ID & $\mathrm{PK}$ & Keywords & $\mathrm{Pl}$ \\
\hline Name & & Excavation report information & $\mathrm{Pl}$, excavation report ID, FK \\
\hline Location & & Investigation agency information & $\mathrm{Pl}$, investigation agency ID, FK \\
\hline Era & & Ruins information & $\mathrm{Pl}$, ruins ID, FK \\
\hline Types & & Relics information & $\mathrm{Pl}$, relics ID, FK \\
\hline Management & & Multimedia information & $\mathrm{Pl}$, multimedia ID, FK \\
\hline Description & & & \\
\hline
\end{tabular}

TABLE 2: Excavation report DB table.

\begin{tabular}{|c|c|c|c|}
\hline Elements & References & Elements & References \\
\hline ID & PK & Language & \\
\hline Title & & Format & \\
\hline Author & & Index & \\
\hline Publisher & & Summary & \\
\hline Date & & Prehistoric remains information & Prehistoric remains ID, FK \\
\hline Types & & Investigation agency information & Investigation agency ID, FK \\
\hline Plate type & & Investigation team & \\
\hline Pages & & Related information & URL \\
\hline
\end{tabular}

TABle 3: Ruins DB table.

\begin{tabular}{|c|c|c|c|}
\hline Elements & References & Elements & References \\
\hline ID & PK & Characteristic & \\
\hline Name & & Excavation report information & $\mathrm{Pl}$, excavation report ID, FK \\
\hline Location & & Prehistoric remains information & Prehistoric remains ID, FK \\
\hline Era & & Relics information & $\mathrm{Pl}$, relics ID, FK \\
\hline Description & & Multimedia information & $\mathrm{Pl}$, multimedia ID, FK \\
\hline Purpose & & Keywords & $\mathrm{Pl}$ \\
\hline Structure & & Management & \\
\hline
\end{tabular}

TABLE 4: Relics DB table.

\begin{tabular}{|c|c|c|c|}
\hline Elements & References & Elements & References \\
\hline ID & $\mathrm{PK}$ & Characteristic & \\
\hline Name & & Excavation report information & $\mathrm{Pl}$, excavation report ID, FK \\
\hline Location & & Prehistoric remains information & Prehistoric remains ID, FK \\
\hline Era & & Ruins information & $\mathrm{Pl}$, ruins $\mathrm{ID}, \mathrm{FK}$ \\
\hline Description & & Multimedia information & $\mathrm{Pl}$, multimedia ID, FK \\
\hline Purpose & & Keywords & $\mathrm{Pl}$ \\
\hline Structure & & Management & \\
\hline
\end{tabular}

TABle 5: Multimedia DB table.

\begin{tabular}{|c|c|c|c|}
\hline Elements & References & Elements & References \\
\hline ID & $\mathrm{PK}$ & Date & \\
\hline Title & & Prehistoric remains information & Prehistoric remains ID, FK \\
\hline Type & & Ruins information & Ruins ID, FK \\
\hline Description & & Relics information & Relics ID, FK \\
\hline Format & & Excavation report information & Excavation report ID, FK \\
\hline Producer & Investigation agency ID, FK & Management & \\
\hline
\end{tabular}

TABLE 6: Investigation agency DB table.

\begin{tabular}{lccc}
\hline Elements & References & Elements & References \\
\hline ID & PK & Contents of investigation & Pl, prehistoric remains ID, FK \\
Name & & Excavation report information & Pl, excavation report ID, FK \\
Description & & Reference & \\
\hline
\end{tabular}




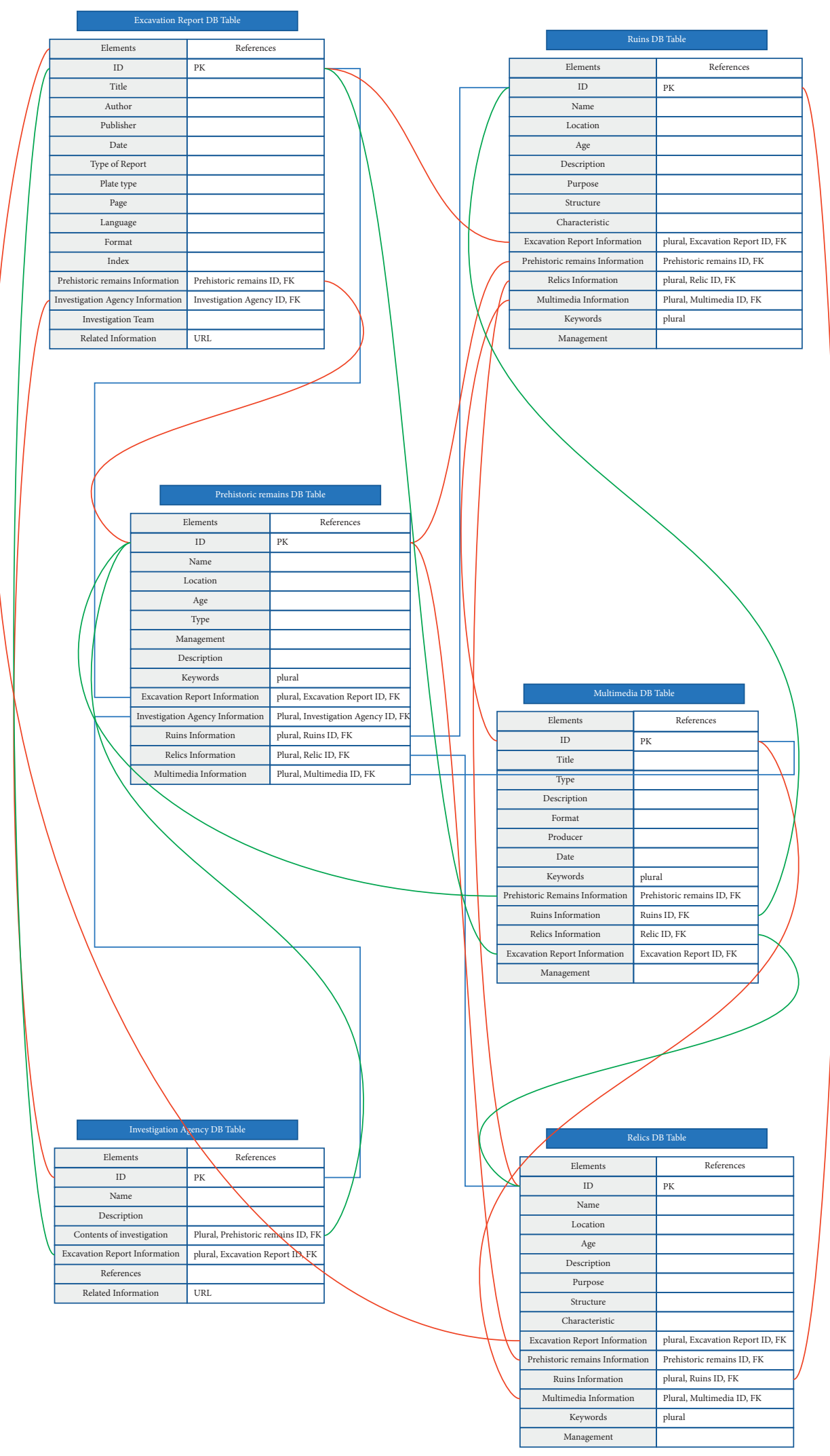

FIgURE 5: A conceptual map of Korean prehistoric remains database. 




Figure 6: Screenshot samples of electronic maps. 


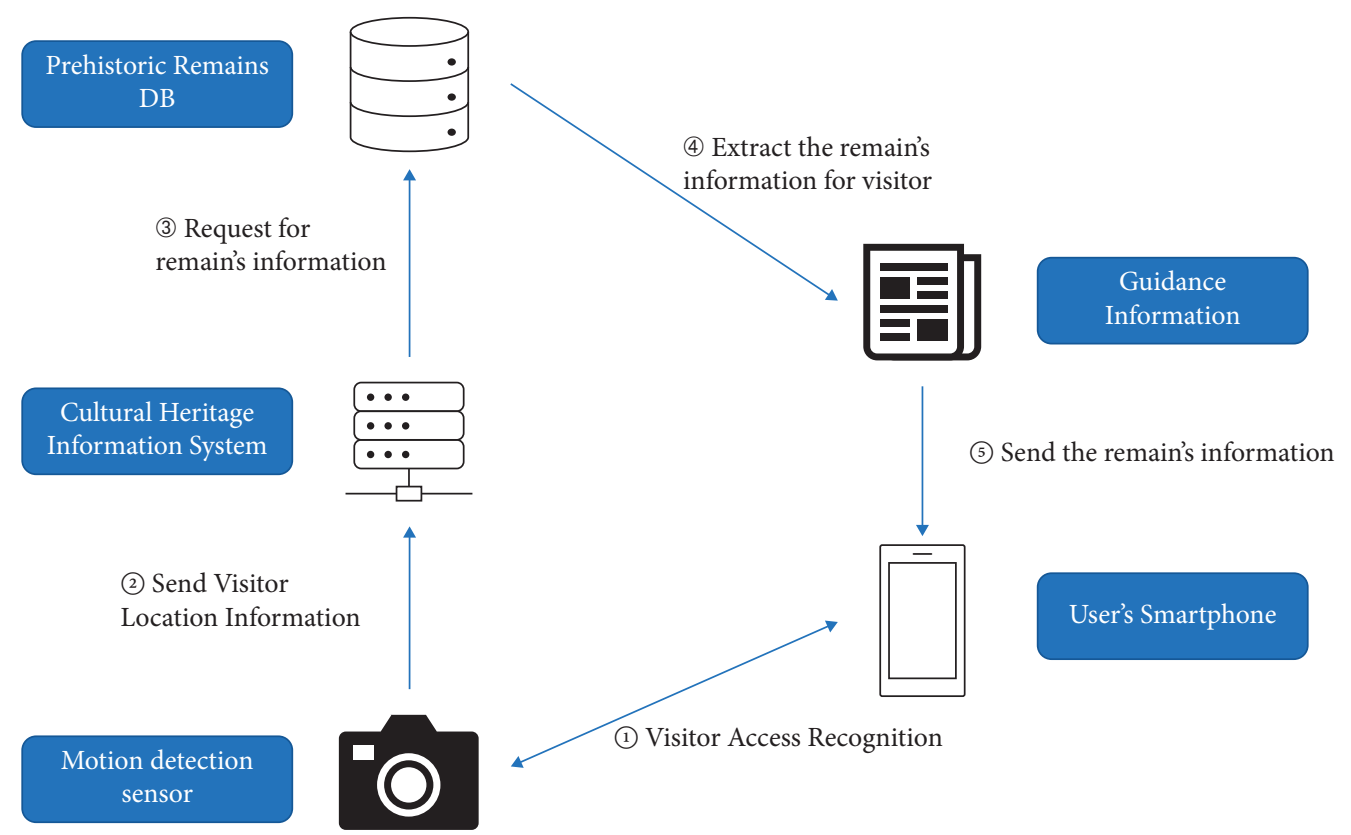

FIGURE 7: Remains information service based on Internet of Things conceptual map.

database. The interconnecting table is shown in the following diagram. (Figure 5).

\section{Conclusions}

There are many ways to collect information about prehistoric remains. It is possible to build a platform of collective intelligence [13] using the popular trend of content curation $[14,15]$ and supplement it using electronic maps as a platform [16]. These methods can be easily processed when various pieces of information related to prehistoric remains are converted into digital data, and the materials are available.

It is an excavation report that contains accurate and abundant information on prehistoric remains. However, it is not easy to obtain information from excavation reports related to prehistoric remains by publishing them in various ways. It may be more difficult for a single entity to collect an excavation report's elements and database it. Therefore, it is necessary to pursue projects at a national level.

A total of 15,378 excavated remains are listed in the excavation timeline of the National Research Institute of Cultural Heritage's research knowledge portal service. This includes not only prehistoric ruins but also those from the early Iron Age, the period of the Three Kingdoms, the Goryeo Dynasty, and the Joseon Dynasty. If you search for the Paleolithic ruins, 409 items are found, as are 351 Neolithic items, 1920 items from the Bronze Age, and 296 items from the Iron Age [17]. Of course, all relics from various eras are included in the search results, but, in general, approximately 2,000 relics can be targeted.

If the Korean prehistoric remains database is promoted in the previously mentioned manner, it can be used in various ways. First, as it is possible to search for prehistoric remains, it is possible to have a macroscopic approach to view prehistoric remains throughout Korea. For example, collective access to the Paleolithic sites in Korea will be available. As a result, the living environment, lifestyle, and cultural characteristics of the Paleolithic Age can be clearly identified. In other words, access from a cultural and historical perspective becomes easy.

In addition, the approach from cultural and processrelated perspectives is also clear. For example, pottery is a representative artifact of the Neolithic and Bronze Ages, which has developed with different characteristics depending on the time and region. Today, only researchers who have studied earthenware for decades are capable of understanding the system or the developmental process. However, when this database is established, they will be able to easily understand the contents as well.

This will provide many other contents. In particular, new remains may be discovered or new cultural implications found through local distribution or the migration of systems. For example, if there is a site of iron production from the early Iron Age, identifying how iron was sourced may also be considered, and in the case of dolmens, which are typical relics of the Bronze Age, efforts can be made to find new relics in tombs and currently unknown residential areas.

Electronic maps are the most useful [18]. Electronic maps have many software tools that are easily accessible to the public. These include Google Maps, Google Earth, and QGIS. If you input the information regarding Korean prehistoric remains into the electronic map platform, the following picture appears: Figure 6.

As with electronic maps, a field where prehistoric remains databases can be used is a cultural heritage information service using the Internet of Things [19]. This service can be divided into two main categories. First, it uses motion-detection sensors. The sensor is attached to the cultural heritage information board and the milestone on the 
cultural heritage viewing route such that it provides the corresponding information through visitors' mobile phones when they stay put or move. The system automatically guides visitors without any additional manipulation, so they can conveniently see the remains. The Internet of Things is provided through the connection of visitors' mobile phone sensors and motion sensors on the cultural heritage information board [20] (Figure 7).

Second, eye-tracking cameras can be used [21]. It is a method of installing a visual tracking camera on the cultural heritage information board to track visitors' reading of information on cultural heritage and automatically guide them with more detailed or additional information. This method has certain limitations. Currently, eye-tracking cameras have problems related to their size and cost. A company recently developed a technology for eye-tracking cameras that can be installed on PC frames, and it is expected to take time to commercialize them. However, these services will be activated and soon be used widely.

These Internet of Things-based services on cultural heritage-related information enable the accumulation of big data to improve cultural heritage management and guidance services by monitoring visitors' viewing patterns and stay time-related information [22]. In addition, more advanced guidance services can be promoted through the use of more advanced sensors. For example, it is possible to check, through sensors on the cultural heritage information board, the height of, estimate the age range of, and provide customized guidance for visitors of each age group [23].

These contents can further enhance the utilization of prehistoric remains information databases. Until now, information related to prehistoric remains has only been gathered separately and individually during the production, distribution, and consumption processes, resulting in many inconveniences. In addition, academically, the analysis of the overall view and relationship of prehistoric remains has been dependent on the individual abilities of researchers. The construction of prehistoric remains databases first contributes to systematically connecting scattered information in a certain context. It is also meaningful to create a foundation for officials and related agencies to collaborate in the process of its establishment. When a database of Korean prehistoric remains information is built and serviced through a single platform, advanced research results will enable experts to overcome these limitations. Moreover, cultural heritage information services, such as electronic map services and the Internet of Things-based information services, will be provided more effectively.

In this way, if an information database on Korea's prehistoric remains is established, it will function as a real digital archive. Individuals or institutions that individually hold information related to prehistoric remains provide archives, which can be structured and contextualized for reuse as resources for distributed databases on digital networks [24]. Information related to prehistoric remains is distributed in various ways, including those through museums, research institutes, government offices, and libraries. By combining them into a single service, knowledge about Korea's prehistoric times is expected to be systematized.

\section{Data Availability}

The data supporting the conclusions of the study are available from the corresponding author.

\section{Conflicts of Interest}

The authors declare no conflicts of interest.

\section{Acknowledgments}

This work was supported by the Ministry of Education of the Republic of Korea, the National Research Foundation of Korea (NRF-2019S1A5B5A02041334), and the National Research Foundation of Korea grant funded by the Korea government (no. 2021S1A5A2A0106408911).

\section{References}

[1] S. M. Nelson, The Archaeology of Korea, Cambridge University Press, Cambridge, UK, 1993.

[2] R. Shao, Y. Q. Guo, X. H. Fu, and S. M. Zhong, "Practice research on the subject knowledge platform based on the knowledge architecture,"vol. 61-66, pp. 2352-5401, in Proceedings of the 2nd Information Technology and Mechatronics Engineering Conference (ITOEC 2016TOEC 2016), vol. 61-66, ITOEC, Chongqing, China, May 2016.

[3] Cultural Heritage Administration, "Excavation reports web service," 2021, https://www.cha.go.kr/html/HtmlPage.do? $\mathrm{pg}=/$ seek/svmnctrdlist03.jsp\&mn=NS_03_13_02.

[4] National Research Institute of Cultural Heritage, "Excavation reports service," 2021, https://portal.nrich.go.kr/kor/original UsrList.do? menuIdx $=565 \&$ bunya_cd $=408$.

[5] National Museum of Korea, "Publication web service," 2021, https://www.museum.go.kr/site/main/archive/report/category/ca tegory_50? $\mathrm{cp}=1$ \&pageSize $=8 \&$ sortOrder=REPORT_YEAR $\% 20$ DESC,\%20ARC_CREATED\&sortDirection=DESC\&catId=50.

[6] K. K. Hirst, "What is an archaeological future? thoughtco," 2020, https://www.thoughtco.com/what-is-an-archaeological-feature170909.

[7] M. Carver, "Excavation methods in archaeology," in Encyclopedia of Global Archaeology, C. Smith, Ed., Springer, New York, NY, USA, 2014.

[8] P. Historical, "Museum commission pennsylvania archeology," 2021, https://www.phmc.state.pa.us/portal/communities/archa eology/resources/value-archaeology.html.

[9] Jigsaw Cambridge shire, "Historic environment record," 2021, https://jigsawcambs.org/images/Introduction_to_Archaeolog ical_Report_Writing.pdf.

[10] Pusan National University Museum, Cultural Remains Survey Report - Gijang-Gun, Pusan National University Museum, Busan, South Korea, 1998.

[11] Ulsan University Museum, Ga-dong Site, Gi-jang I, Ulsan University Museum, Ulsan, South Korea, 2008.

[12] Bu Kyung Cultural Relics Research Institute, Gadong Historic Site and Gijang Bangok-Ri Historic Site I. II. III, Bu Kyung Cultural Relics Research Institute, Busan, South Korea, 2014.

[13] J. M. Leimeister, "Collective intelligence," Business \& Information Systems Engineering, vol. 2, no. 4, pp. 245-248, 2010.

[14] E. Yakel, "Digital curation," OCLC Systems and Services: International digital library perspectives, vol. 23, no. 4, pp. 335-340, 2007. 
[15] Y. Feng and L. Richards, "A review of digital curation professional competencies: theory and current practices," Records Management Journal, vol. 28, no. 1, pp. 62-78, 2018.

[16] M. K. Buckland and L. R. Lancaster, "Advances in discovery: the electronic cultural atlas initiative experience," First Monday, vol. 11, no. 8, 2006.

[17] National Research Institute of Cultural Heritage, "The excavation timeline," 2021, https://portal.nrich.go.kr/kor/ excavationChronologyUsrList.do?menuIdx $=566$.

[18] J.-H. Kang and S.-H. Moon, "Research on knowledge map using electronic cultural atlas," Journal of the Korea Institute of Information and Communication Engineering, vol. 18-6, no. 1383 , pp. 2234-4772, 2014.

[19] M. Gigli and S. Koo, "Internet of things: services and applications categorization," Advances in Internet of Things, vol. 1, no. 2, pp. 27-31, 2011.

[20] J. W. Kim, S. H. Sul, and J. B. Choi, "Development of real-time internet of things motion detection platform applying noncontact sensor based on open source hardware," International Journal of Distributed Sensor Networks, vol. 16, no. 7, Article ID 155014772094402, 2020.

[21] A. F. Klaib, N. O. Alsrehin, W. Y. Melhem, H. O. Bashtawi, and A. A. Magableh, "Eye tracking algorithms, techniques, tools, and applications with an emphasis on machine learning and internet of things technologies," Expert Systems with Applications, vol. 166, pp. 114037-114174, 2021.

[22] M. Ge, H. Bangui, and B. Buhnova, "Big data for internet of things: a survey," Future Generation Computer Systems, vol. 87, no. 601-614, pp. 601-614, 2018.

[23] Z. Zheng, J. Zhu, and M. R. Lyu, "Service-generated big data and big data-as-a-service: an overview," in Proceedings of the 2013 IEEE International Congress on Big Data, BigData, vol. 403-410, July 2013.

[24] K. Ezéchiel, S. Kant, and R. Agarwal, "A systematic review on distributed databases systems and their techniques," Journal of Theoretical and Applied Information Technology, vol. 96, no. 1, pp. 233-266, 2019. 